Meta

Journal des tradlucteurs

Translators' Journal

\title{
Recherche documentaire dans le cadre d'une recherche thématique
}

\section{Michèle Valiquette}

Volume 24, numéro 3, septembre 1979

URI : https://id.erudit.org/iderudit/004524ar

DOI : https://doi.org/10.7202/004524ar

Aller au sommaire du numéro

Éditeur(s)

Les Presses de l'Université de Montréal

ISSN

0026-0452 (imprimé)

1492-1421 (numérique)

Découvrir la revue

Citer cet article

Valiquette, M. (1979). Recherche documentaire dans le cadre d'une recherche thématique. Meta, 24(3), 354-361. https://doi.org/10.7202/004524ar d'utilisation que vous pouvez consulter en ligne.

https://apropos.erudit.org/fr/usagers/politique-dutilisation/ 


\section{RECHERCHE DOCUMENTAIRE DANS LE CADRE D'UNE RECHERCHE THÉMATIQUE}

- La recherche documentaire est la première étape de toute recherche terminologique thématique, et sans contredit la plus importante. En effet, les terminologues les plus compétents ne peuvent donner aucune assurance de la qualité de leur travail s'ils ne peuvent s'appuyer sur une documentation de base solide, étayée par nombre de textes spécialisés.

C'est le terminologue lui-même qui doit effectucr la recherche des documents nécessaires à la bonne marche de son travail. Dans le cas d'un stage de formation, le moniteur ou chef d'équipe guide le stagiaire dans cette recherche documentaire.

Le terminologue s'assure, dans la mesure du possible, la collaboration des bibliothécaires afin qu'ils lui indiquent toutes les sources d'information qui pourraient lui être utiles et qu'ils lui fournissent tous les renseignements pertinents qu'ils pourraient recueillir sur la documentation disponible, récemment parue ou à paraître, dans le domaine concerné ${ }^{\star}$. »

3. Id., ibid., p. 80 .

1. Secrétariat d'Etat, Bureau des traductions, Direction générale de la terminologie et de la documentation, Description de l'Acte de terminologie, rédigé par Michèle Cossette, 1976 , p. 2. 


\section{Types d'étude effectuée en recherche thématique}

Le terminologue peut effectuer deux types d'étude en recherche thématique :

a) Etude du vocabulaire de base d'un domaine : c'est en général le cas lors d'une première thématique. La documentation est surtout constituée d'ouvrages spécialisés.

b) Étude du vocabulaire de pointe d'un domaine : on trouve du vocabulaire de pointe dans les domaines techniques. La documentation doit être très récente. Elle est surtout constituée de périodiques.

\section{Critères intervenant dans le choix d'un sujet de thématique}

a) Besoins des clients (en général les traducteurs).

b) Nouveauté du sujet (en vue de l'emmagasinement en banque de fiches portant sur des domaines nouveaux).

c) Disponibilité de la documentation (c'est en général le critère qui prévaut).

A documentation égale, entre un sujet qui satisfait aux deux premiers critères et un qui n'y satisfait pas, choisir le premier.

\section{Délimitation du sujet de la thématique}

"Le terminologue doit [...] effectuer, à la suite de lectures générales, un découpage sommaine du sujet afin de ne pas laisser de côté, à cette étape, des sous-domaines ou des domaines connexes dont il aurait pu, à première vue, ne pas soupçonner l'importance ou l'utilité ; afin, également, d'élargir le champ des sources possibles de documentation ${ }^{2}$.

Donc, à l'aide des encyclopédies anglaises et françaises, délimiter le sujet choisi de la façon suivante :

a) Identification des principaux sous-domaines dans chacune des langues. Les titres de paragraphes correspondent souvent aux principaux sous-domaines.

b) Recoupement des sous-domaines traités à la fois dans les deux langues. matique.

c) Choix du (ou des) sous-domaine(s) qui fera(ront) l'objet de la thé-

\section{Repérage de la documentation au bureau}

a) Recenser les articles d'encyclopédie, les ouvrages spécialisés, les périodiques et les normes que possède le centre de documentation du bureau sur le sujet de recherche.

Le terminologue doit prendre note des titres utiles. Il peut rédiger des fiches bibliographiques (une fiche par ouvrage) ou dresser une liste des ouvrages. Pour chacun des titres doivent figurer les données suivantes :

2. M. Cossette, op. cit., p. 3. 
- nom de l'auteur,

- titre (pour les périodiques : «titre de l'article » in «titre du périodique »),

- volume (s'il y a lieu),

- numéro, maison et lieu d'édition,

- année (pour les périodiques : mois et année),

- pages ou chapitres précis se rapportant au sujet,

- mention de traduction (s'il y a lieu).

b) Constituer soi-même une bibliographie à partir des bibliographies qui figurent en fin d'articles dans les encyclopédies. Les ouvrages cités sont souvent excellents, et ceux dont les titres reviennent d'un article à l'autre sont généralement de bons ouvrages de base ou des ouvrages qui font autorité dans le domaine.

Consulter les bibliographies figurant en fin des ouvrages spécialisés. Ne pas oublier les numéros des Techniques de l'ingénieur que possède la bibliothèque et qui se rapportent au sujet de la thématique. Il y figure souvent une imposante bibliographie. Consulter aussi les catalogues de normes que possède la bibliothèque afin d'y repérer les normes se rapportant au sujet.

Particularités des encyclopédies ${ }^{3}$ :

ENSCI, AMERI, ENBRI : bibliographie en anglais ;

UNIVE : bibliographie en anglais et en français ;

GRENB : bibliographie en anglais et en français + mention des ouvrages traduits ; AUTEC : pas de bibliographie.

\section{Repérage de la documentation à l'extérieur du bureau}

\section{a) Bibliothèques universitaires ou autres}

«Le fait de se rendre dans les bibliothèques et les centres de documentation [...] permet de voir ce qu'ils possèdent et de se rendre compte de la valeur terminologique de certains volumes, de certains périodiques, même de certaines collections. On évite ainsi, dans la mesure du possible, de commander des ouvrages qui, à l'usage, s'avèrent inutiles du point de vue terminologique ${ }^{4}$. \$

\section{1) Fichiers}

Consulter le fichier auteurs-titres d'une bibliothèque universitaire ou autre afin d'y repérer les ouvrages inscrits sur la liste (ou les fiches) bibliographique(s). Consulter aussi le fichier sujets pour compléter cette liste ou ces fiches.

\section{2) Catalogues de périodiques}

Dans le cas d'une thématique qui porte sur le vocabulaire de pointe d'un domaine, la documentation sera surtout constituée de périodiques comme nous

\footnotetext{
3. AMERI The Encyclopaedia Americana.

AUTEC Encyclopédie internationale des sciences et des techniques.

ENBRI Encyclopaedia Britannica.

ENSCI McGraw-Hill Encyclopedia of Science and Technology.

GRENB La Grande Encyclopédie Larousse en 20 volumes.

4. M. Cossette, op. cit., p. 5.
} 
l'avons dit. Recenser les articles de périodiques traitant du sujet de recherche. Pour le français, consulter les index Périodex et Radar et pour l'anglais, les Abstracts.

Les index Périodex et Radar sont des mensuels qui font l'objet d'une refonte annuelle. C'est cette refonte annuelle qu'il faut consulter. Les articles de revue y sont regroupés par sujet.

Les Abstracts sont classés par grands domaines (Sociological Abstracts, Psychological Abstracts, etc.). Ils constituent une source très précieuse car les articles y sont résumés. Le terminologue sait tout de suite s'ils font l'affaire ou non. Il n'a pas à consulter les revues.

\section{3) Bulletins signalétiques et thésaurus}

"Repérer dans les bulletins signalétiques (tels ceux du CNRS) et les thésaurus les articles de périodiques et les chapitres des ouvrages portant sur le sujet à l'étude, et qui semblent d'un intérêt particulier ${ }^{5}$. \$

\section{b) Entreprises}

* Il est important de se rendre dans les entreprises, surtout les plus importantes, qui cuvrent dans le domaine qu'on veut étudier. Beaucoup d'entre elles s'intéressent à la question de la terminologie. $Y$ aller et expliquer aux personnes intéressées le but de la recherche et l'avantage qu'elles pourraient en tirer peut susciter une collaboration fructueuse. Il est parfois possible d'avoir accès à la documentation interne de l'entreprise : c'est dans ces textes, qui ne seront jamais publiés, qu'on trouve le vocabulaire de pointe. On peut aussi, au cours d'un entretien, recueillir des renseignements précieux sur la valeur, au niveau du fond, des publications relatives au domaine à l'étude ${ }^{6}$.

\section{Evaluation de la documentation}

Dans le cas d'une thématique, cette évaluation se fait en général à priori, c'est-à-dire sans bien connaître les ouvrages du fait qu'on ne les a jamais utilisés. Cette évaluation se fait sur les lieux mêmes de la bibliothèque extérieure et permet de distinguer les bons ouvrages des autres. Elle évite au terminologue d'emprunter par la suite des ouvrages qui ne conviennent pas.

a) Types de documentation

1) Ouvrages lexicographiques et terminologiques (dictionnaires)

2) Ouvrages non lexicograhiques

- à intention pédagogique (manuels et notices techniques)

- à intention informative (études, monographies, articles de journaux ou de revues) ${ }^{7}$

5. M. Cossette, op. cit., p. 4.

6. Ibid., p. 5.

7. Robert Dubuc, Manuel pratique de terminologie, Montréal, Linguatech, 1978, p. 90-91. 


\section{b) Critères d'évaluation de la documentation}

\section{1) Ouvrages lexicographiques et terminologiques}

On peut utiliser les trois critères suivants :

$1^{\circ}$ \&Indications méthodologiques. - Il est important, pour se faire une juste idée du sérieux et de la portée d'un ouvrage, d'avoir des indications sur la façon dont l'auteur a élaboré son travail. Comment a-t-il établi sa bibliographie? A-t-il indiqué ses domaines d'emploi? Les synonymes sont-ils différenciés et comment ${ }^{8}$ ? 》

$2^{\circ}$ «La structure de l'ouvrage constitue également un critère d'évaluation important. Comment les notions sont-elles systématisées? Comment sont structurés les renvois ? Comment l'auteur a-t-il délimité ses entrées? $\mathrm{Y}$ a-t-il un index des mots cités hors de l'ordre alphabétique normal ${ }^{9}$ ?

$3^{\circ}$ \& Enfin comme troisième critère, on pourrait retenir la qualité de la présentation : cohérence typographique, conventions, illustrations, etc. ${ }^{10} \gg$

\section{2) Ouvrages non lexicographiques}

$1^{\circ}$ Date de publication. - \& L'année de publication est un facteur important, surtout dans les domaines, comme l'informatique, où les techniques évoluent très vite, entraînant ainsi des créations ou des modifications fréquentes du vocabulaire. Cependant, comme le vocabulaire de base, en général, varie peu, l'âge des volumes consultés en pareil cas importe moins ${ }^{11}$. 》

De façon plus précise, il faut utiliser des ouvrages ou des périodiques très récents (moins de cinq ans) pour une thématique portant sur le vocabulaire de pointe. Pour le vocabulaire de base, des ouvrages qui ont vingt ans ou même trente ans d'existence peuvent faire l'affaire.

Chose certaine, la documentation doit être à peu près contemporaine dans les deux langues. Par exemple, il faut éviter d'utiliser en anglais un ouvrage publié en 1960 et en français un ouvrage qui date de 1920. L'écart est alors trop grand ce qui peut rendre difficile, voire impossible, les recoupements d'une langue à l'autre.

$2^{\circ}$ Indications méthodologiques. - Elles sont en général données dans l'introduction, la préface et la table des matières de l'ouvrage. Elles permettent de se faire une idée juste du sérieux et de la portée d'un ouvrage.

- L'auteur a-t-il suivi une méthode? L'explique-t-il clairement dans son introduction?

- L'origine géographique de l'ouvrage est-elle bien identifiée dans l'introduction?

- Le sujet est-il bien découpé ? La table des matières est-elle détaillée ?

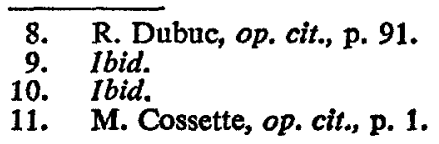


- L'auteur jouit-il d'une certaine autorité dans son domaine? Quelle est sa crédibilité ? Emploie-t-il une terminologie rigoureuse ou bien utilise-t-il une avalanche de synonymes? La lecture de la préface et le survol de quelques chapitres peuvent renseigner sur ce point. \&Il est bon de savoir, dans la mesure du possible, quel est le degré de « crédibilité » de l'auteur dans sa spécialité. Dans les domaines de pointe, par exemple, il faut se méfier des «terminologies personnelles \ crées et employées, unilatéralement et plus ou moins arbitrairement, par certains auteurs. Ces termes ne sont pas nécessairement mauvais, mais il importe de ne pas les considérer comme des termes admis par l'usage ${ }^{12}$.

- La bibliographie est-elle exhaustive?

- Y a-t-il un index qui permette de repérer rapidement les termes?

En général, les ouvrages à intention pédagogique tels que les manuels de classe sont de bons ouvrages car ils définissent clairement les notions.

$3^{\circ}$ Public-cible. - II faut [...] tenir compte du public-cible de l'ouvrage. Ce dernier a-t-il été rédigé dans une optique de vulgarisation, d'enseignement ou de haute spécialisation? $\mathrm{La}$ langue utilisée sera différente dans chaque cas $^{13}$.

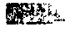

Il vaut mieux choisir des ouvrages qui ne sont ni trop vulgarisés ni trop techniques. Entre les deux, toutefois, les ouvrages plus techniques conviennent davantage à une recherche thématique que les ouvrages vulgarisés car ces derniers omettent parfois des nuances importantes ou expliquent de façon trop simpliste des phénomènes complexes ce qui peut fausser les notions ou du moins n'aide pas à les clarifier.

$4^{\circ}$ Langue de rédaction. - « Les ouvrages mal écrits dans leur langue sont évidemment considérés au départ comme « peu fiables». Cependant, même des ouvrages très bien rédigés peuvent contenir des termes plus ou moins douteux : c'est le cas, par exemple, en gestion, où même les auteurs européens abusent des calques et des emprunts. [...] Au départ sont étiquetés «à éviter » les ouvrages traduits et les dictionnaires bilingues ou plurilingues. On peut certes les utiliser, mais il importe de toujours vérifier l'exactitude des termes qu'on y trouve, dans des ouvrages en langue originale ${ }^{14}$.

\section{Commande ou emprunt de documents}

a) «Indiquer au service de documentation les ouvrages et les périodiques ou numéros de périodiques retenus pour qu'il les commande aux maisons d'édition concernées.

b) Profiter, dans la mesure du possible, des prêts entre bibliothèques et centres de documentation.

c) Ecrire aux entreprises (surtout étrangères) pour obtenir d'elles toute la documentation qu'elles peuvent envoyer.

12. M. Cossette, op. cit., p. 6.

1.3. Ibid., p. 2.

14. Ibid. 
d) Photocopier ou faire photocopier, dans les bibliothèques, centres de documentation et entreprises, des chapitres, des parties d'ouvrages et des articles afin d'éviter de commander ou d'emprunter l'ouvrage dans son ensemble. Ceci vaut aussi pour les périodiques, que les bibliothèques ne prêtent jamais, et qu'il est parfois impossible d'obtenir de l'éditeur (par exemple, numéros épuisés, etc.) ${ }^{15}$.

Pour que le service de documentation puisse procéder de façon efficace à la commande ou à l'emprunt d'ouvrages, le terminologue doit donner la référence bibliographique complète de chaque ouvrage et de chaque périodique comme indiqué au point 4 .

Le prêt entre bibliothèques et la photocopie sont des systèmes qui fonctionnent bien. La photocopie permet d'obtenir sur-le-champ la documentation nécessaire. Il faut compter de trois jours à trois semaines pour obtenir les ouvrages par prêt entre bibliothèques. Tout dépend de la localisation de la bibliothèque prêteuse.

Les commandes d'ouvrages sont soumises à certains aléas. Elles dépendent en effet du budget alloué au centre de documentation et les délais de réception des ouvrages sont souvent très longs (souvent plus de six mois, parfois même un an).

Quant aux échanges épistolaires avec les entreprises, les délais varient. Tout dépend de l'entreprise.

\section{Composition du corpus documentaire}

\section{a) Documentation rassemblée}

* La documentation [...] réunie doit comprendre des ouvrages de base, des ouvrages spécialisés, des périodiques, des manuels techniques, des dictionnaires et des vocabulaires techniques, généraux et spécialisés, bilingues pour les équivalences, unilingues pour les définitions ou la précision de contextes définitoires, explicatifs ou d'utilisation, des encyclopédies, des textes publicitaires, des documents d'entreprise, des normes, etc., sans oublier les dictionnaires généraux ${ }^{16}$. Pour faire une thématique valable, il faut utiliser au moins deux bons ouvrages spécialisés dans chaque langue si on veut avoir un certain choix parmi les justifications et être en mesure de régler les problèmes terminologiques qui se présentent. Par contre, le terminologue ne devrait pas avoir à sa disposition plus de quatre bons ouvrages spécialisés dans chaque langue s'il veut s'y retrouver. Un trop grand choix engendre la confusion.

Une fois la documentation rassemblée, le terminologue procède sur-le-champ a la demande des codes de source par téléphone et par écrit.

b) Index des termes emmagasinés

"Étant donné que le fichier TERMIUM ${ }^{17}$ a déjà un capital important de fiches en mémoire, on doit éviter, dans la mesure du possible, le dédoublement inutile

15. M. Cossette, op. cit., p. 5.

16. Ibid., p. 6.

17. TERMIUM : Nom du fichier terminologique de la banque de terminologie du Secre 
de ces fiches. $\AA$ cette fin, un autre document est indispensable au terminologue chercheur. II s'agit d'un index des termes déjà emmagasinés et qui appartiennent au domaine dont on entreprend l'étude. Ce relevé est fait automatiquement au moyen du code du domaine en question ${ }^{18}$, ,

\section{Consultation de spécialistes}

- Le terminologue doit, dans la mesure du possible, établir une liste de spécialistes du sujet à l'étude, qu'il s'agisse de traducteurs, de techniciens ou de professionnels du domaine en question, à qui il pourra s'adresser s'il a un problème terminologique particulièrement difficile à résoudre, ou encore, s'il a besoin d'informations techniques ${ }^{19}$.

S'il a effectué la recherche documentaire avec soin, le terminologue dispose alors de tous les éléments qui lui permettront de s'attaquer sérieusement à la recherche thématique.

MichÈle VALiQuetTe 